\title{
Percepción estudiantil de un curso universitario que integró los recursos de Google como apoyo educativo
}

\author{
Student Perception of the Evaluation of a University Course Integrating \\ Google Resources as Educational Support
}

\section{Percepção do aluno sobre um curso universitário que integrou recursos do Google como suporte educacional}

\author{
Arturo González Torres \\ Centro de Estudios e Investigaciones para el Desarrollo Docente CENID A.C. México \\ Tecnológico Nacional de México, Campus Milpa Alta, México \\ cann.azteca13@gmail.com \\ https://orcid.org/0000-0002-3337-7600
}

\section{Resumen}

Actualmente, dadas las exigencias del mundo actual, se está experimentado un cambio en la forma de aprender y de enseñar. Los implicados en el proceso educativo se han visto en la labor de incorporar las tecnologías de la información y la comunicación (TIC) en la educación. El objetivo de este estudio fue conocer la percepción estudiantil de un curso universitario que integró los recursos de Google como apoyo educativo. Para ello, se realizó un estudio de carácter descriptivo enfocado en una investigación de campo. La recolección de la información se hizo mediante un cuestionario estructurado con 36 preguntas, que fueron evaluadas con una escala de Likert del uno al cinco, más dos preguntas adaptadas a 27 estudiantes. La información obtenida fue procesada con ayuda del programa informático Microsoft Excel. Los resultados muestran que el grupo de alumnos que cursó la asignatura manifestó gran motivación en el desarrollo de las actividades programadas, al igual que un gran interés por aprender cada una de las herramientas de Google que integró la materia. Se concluye que, debido a su facilidad de uso, acceso gratuito y características, los escolares 

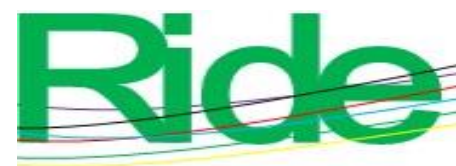

Revista Iberoamericana para la Investigación y el Desarrollo Educativo ISSN $2007-7467$

percibieron de forma positiva la integración de los recursos de Google como apoyo educativo en la asignatura. Los recursos que más destacaron fueron Documentos, Formularios, Tasks, Sites y Gmail.

Palabras clave: educación superior, formación universitaria, Google, valoración.

\begin{abstract}
Currently, given the demands of today's world, a change is being experienced in the way of learning and teaching. Those involved in the educational process have seen themselves in the work of incorporating information and communication technologies (ICT) in education. The objective of this study was to know the student perception of a university course that integrated Google resources as educational support. For this, a descriptive study was carried out focused on a field investigation. The information was collected through a structured questionnaire with 36 questions, which were evaluated with a Likert scale from one to five, plus two questions adapted to 27 students. The information obtained was processed with the help of the Microsoft Excel computer program. The results show that the group of students who took the subject showed great motivation in the development of the programmed activities, as well as a great interest in learning each of the Google tools that integrated the subject. It is concluded that, due to its ease of use, free access and characteristics, schoolchildren positively perceived the integration of Google resources as educational support in the subject. The resources that stood out the most were Documents, Forms, Tasks, Sites and Gmail.
\end{abstract}

Keywords: higher education, university education, Google, valuation. 


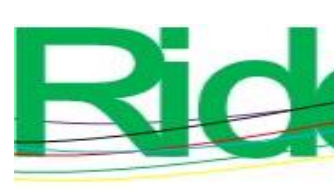

Revista Iberoamericana para la Investigación y el Desarrollo Educativo ISSN $2007-7467$

\section{Resumo}

Atualmente, diante das demandas do mundo atual, está ocorrendo uma mudança na forma de aprender e de ensinar. Os envolvidos no processo educacional têm se envolvido no trabalho de incorporação das tecnologias de informação e comunicação (TIC) na educação. O objetivo deste estudo foi conhecer a percepção dos alunos sobre um curso universitário que integrou recursos do Google como suporte educacional. Para isso, foi realizado um estudo descritivo com foco em uma investigação de campo. As informações foram coletadas por meio de um questionário estruturado com 36 questões, as quais foram avaliadas em uma escala Likert de um a cinco, mais duas questões adaptadas para 27 alunos. As informações obtidas foram processadas com auxílio do programa de computador Microsoft Excel. Os resultados mostram que o grupo de alunos que cursou a disciplina demonstrou grande motivação no desenvolvimento das atividades programadas, bem como grande interesse em aprender cada uma das ferramentas do Google que integram a disciplina. Conclui-se que, pela facilidade de uso, livre acesso e características, os escolares perceberam positivamente a integração dos recursos do Google como suporte educacional na temática. Os recursos que mais se destacaram foram Documentos, Formulários, Tarefas, Sites e Gmail.

Palavras-chave: ensino superior, ensino universitário, Google, avaliação.

Fecha Recepción: Mayo 2020

Fecha Aceptación: Marzo 2021

\section{Introducción}

Para poder hacer frente a las exigencias actuales en el ámbito educativo y laboral no solo basta con dar cabida a las herramientas tecnológicas, sino que también hay que encontrar la mejor manera de utilizarlas en cada contexto. Las tecnologías de la información y la comunicación (TIC) son capaces de promover un aprendizaje más eficaz y mucho más económico que la educación presencial. En el Instituto Tecnológico de Milpa Alta (Ciudad de México), donde se llevó a cabo el presente proyecto, la parte directiva está comprometida con el cuidado del medio ambiente y los recursos que se utilizan en el proceso de enseñanza. Esto ha provocado que, en el proceso educativo, los jefes académicos soliciten a los docentes el mayor cuidado en los recursos: copias, exámenes y portafolios de evidencia. Para ello, se han impulsado diversas estrategias que van desde el almacenamiento en la nube para el portafolio de evidencias (Dropbox) y utilizar sistemas para la gestión del aprendizaje (LMS) 


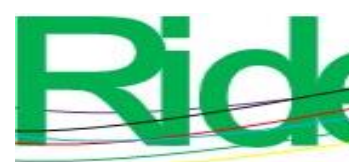

Revista Iberoamericana para la
Investigación y el Desarrollo Educativo
ISSN $2007-7467$

para la realización de exámenes hasta implementar plataformas para la gestión de reuniones de manera sincrónica (por ejemplo, Teams) o el uso de blogs para compartir información con los alumnos. Asimismo, todos los docentes poseen una cuenta institucional en Gmail, y con ella muchas de las herramientas educativas de Google. Sin embargo, son pocos los docentes que conocen los beneficios de tener este servicio; la mayoría solo la utilizan para enviar y recibir información por correo electrónico. Esto es precisamente debido al desconocimiento del abanico de herramientas que Google ofrece en el ámbito de la educación.

Teniendo en cuenta lo anterior, surgió la idea de la presente investigación: conocer la percepción estudiantil de un curso universitario que integra los recursos de Google como apoyo educativo. Y gracias al respaldo del Instituto Tecnológico de Milpa Alta, la idea se materializó y pudo ser aplicada con alumnos que cursaron la materia Gestión de la Producción II, que forma parte del programa de ingeniería en Gestión Empresarial. Así pues, el objetivo principal fue conocer la percepción y evaluación estudiantil de un curso universitario que integró las herramientas de Google como apoyo educativo. Además, tangencialmente, examina, a través de la lente de los escolares, la potencialidad de las herramientas de Google en el ámbito educativo. Cabe señalar que la investigación se llevó a cabo durante el curso del posdoctorado de Tecnología Educativa en el Centro de Estudios e Investigaciones para el Desarrollo Docente (Cenid).

Finalmente, siguiendo a Brodersen y Mellluzo (2017), vale la pena advertir que en la época actual un aprendizaje combinado (blended learning) solo es posible si se logra una sinergia entre las clases tradicionales en el aula y el aprendizaje electrónico.

\section{Incursión de las TIC en la educación}

Hay varios esfuerzos que han documentado la implementación de las TIC en el ámbito educativo y el impacto que han causado. Por ejemplo, Claro (2010) desarrolló un estudio sobre el efecto que tienen las TIC en la enseñanza de los alumnos. Como conclusión, el autor destaca que para obtener resultados positivos en el aprendizaje las condiciones de acceso a las TIC deberán de ser las adecuadas. Rodríguez (2010), por su parte, realizó una investigación sobre la relación existente entre el empleo de las TIC y la enseñanza en la universidad. Aquí se destaca que, para obtener cambios positivos en la educación, tanto los profesores como el uso de las TIC y las estrategias de enseñanza deben ir encamindos hacia un mismo fin. Igualmente, Ávila y Riascos (2011) midieron el impacto de las TIC en los 


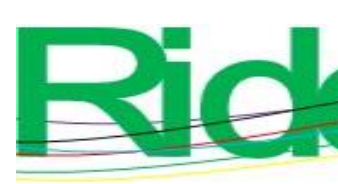

Revista Iberoamericana para la
Investigación y el Desarrollo Educativo
ISSN $2007-7467$

estas nuevas tecnologías. Grájeda (2015) realizó una investigación cuyo propósito fue demostrar si los alumnos desarrollan un mejor aprendizaje al usar la Web 2.0. Al final, el autor subraya sobre todo el aprendizaje colaborativo. Vale la pena mencionar también el trabajo de Rodríguez (2015), quien evaluó el beneficio que se tiene al emplear las TIC con estudiantes con discapacidad intelectual.

Finalmente, los estudios de Gómez, Contreras y Gutiérrez (2016), Plascencia y Beltrán (2016), Esparza (2017), García-Valcárcel y Tejedor (2017), Hernández (2017) y Mejía y Gómez (2017), si bien cada uno tiene sus peculiaridades, al final se inclinan hacia la misma dirección: el impacto positivo que genera la implementación de las TIC en las aulas.

\section{Estudios previos de uso de Google en el ámbito educativo}

López, Ledesma y Escalera (2009) mencionan que los elementos y consideraciones a tener en cuenta en un ambiente virtual de aprendizaje son la interacción, confianza, accesibilidad y la motivación. Gómez (2011) valoró en particular el efecto que tiene el uso de Google Earth con los alumnos de primer grado de educación secundaria. El trabajo finiquita que los estudiantes de primer grado, en su mayoría, presentan resultados positivos al usar este recurso. De acuerdo con González (2011), las herramientas Docs, Groups y Académico de Google son de gran ayuda durante para la práctica docente. Castellanos y Martínez (2013), por su parte, midieron la experiencia de los estudiantes al participar en una actividad colaborativa utilizando Google Drive. Los resultados que se obtuvieron fueron, en primer lugar, la facilidad del manejo de este recurso, y en segundo, lo sencillo que resultó la formación y participación de grupos de trabajo en línea. Galantini (2015) desarrolló un estudio cuya finalidad fue emplear la herramienta Site de Google en el aprendizaje de estudiantes de posgrado. Y encontró una correlación significativa entre el uso de la herramienta y las siguientes variables: distribución de libros virtuales, planificación de foros virtuales y organización de cuadernos virtuales

Al igual que lo que sucede en los casos anteriores, hay una lista extensa de trabajos que concluyen en la utilidad de las herramientas educativas de Google (Gmail, Drive, Docs, Sites, Forms, etc.) a lo largo del proceso de enseñanza-aprendizaje y el beneficio que traen para cada uno de los actores involucrados en este: directivos, profesores y alumnos (Almeida, Chuco y Lavado, 2015; Barrios, 2017; Barrios y Casadei, 2014; Delgado y Casado, 2013; 


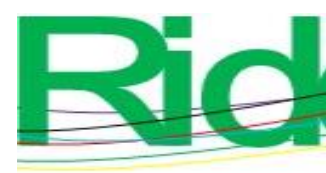

Revista Iberoamericana para la
Investigación y el Desarrollo Educativo
ISSN $2007-7467$

Huzco y Romero, 2018; Quinatoa, 2015; Martínez, 2016; Roda y Sassano, 2016; Suárez, 2018; Tolosa y García, 2011; Vilela, Purihuamán y Nuñez, 2016; Zambrano, 2015).

\section{Materiales y método}

\section{Tipo de estudio}

Se trata de una investigación de carácter descriptivo. Además, se concibió como transeccional descriptiva, ya que se realizó en un único momento en el tiempo sobre un grupo determinado (Hernández, Fernández y Baptista, 2003).

\section{Participantes}

La población de la investigación estuvo integrada por los alumnos de la materia de Gestión de la Producción II, la cual forma parte de las asignaturas del sexto semestre de la ingeniería en Gestión Empresarial del Instituto Tecnológico de Milpa Alta. El número de estudiantes inscritos y tomados en cuenta para la investigación fue de 27 escolares.

\section{Instrumento}

Para medir la calidad del curso, se utilizó y adaptó el cuestionario de Santoveña (2010). Dicho cuestionario está organizado en tres dimensiones principales: 1) Calidad general del entorno y de la metodología didáctica (del ítem 1 al 17), 2) Calidad técnica: navegación y diseño (del ítem 18 al 26) y 3) Calidad técnica: recursos multimedia (del ítem 27 al 36). En cuanto a la fiabilidad, la primera dimensión tuvo un valor de alfa de Cronbach de 0.945 , la segunda de 0.932 y la tercera de 0.924 . Cabe señalar que al cuestionario en cuestión se le agregaron dos ítems más: uno de ellos evaluó el conocimiento previo de los recursos de Google y el segundo midió el grado de satisfacción de cada herramienta de Google utilizada en la materia. Para el caso de este par de ítems, se revisaron y adaptaron las preguntas del estudio de Delgado y Casado (2013). 


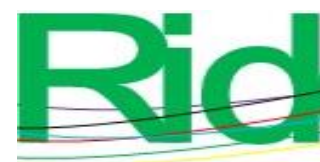

Revista Iberoamericana para la Investigación y el Desarrollo Educativo ISSN 2007 - 7467

\section{Procedimiento}

\section{Fase cero}

Se estudió el programa de estudio de la materia con el fin de conocer los temas, las actividades de aprendizaje y las competencias que la asignatura dicta. También se llevó a cabo un análisis de los recursos de Google con las actividades que se requieren incorporar para lograr un aprendizaje significativo en los estudiantes. Por último, se realizaron dos diagnósticos: el primero fue de las herramientas de Google y el segundo fue para conocer el uso que le dan los alumnos a las TIC.

\section{Fase uno}

Se elaboró el esbozo de la asignatura seleccionada (figura 1). Para ello, se utilizó el recurso Google Sites, que sirvió de apoyo a la materia. y en el que se incluyeron las herramientas seleccionadas en la etapa anterior. Asimismo, se diseñaron las actividades de aprendizaje del curso, poniendo especial atención en el modo de secuenciar y organizar el contenido. Se utilizó el bosquejo de la plantilla clásica de Site, la cual es predeterminada y fue creada por Montañana (2017).

Figura 1. Plantilla predeterminada de Sites

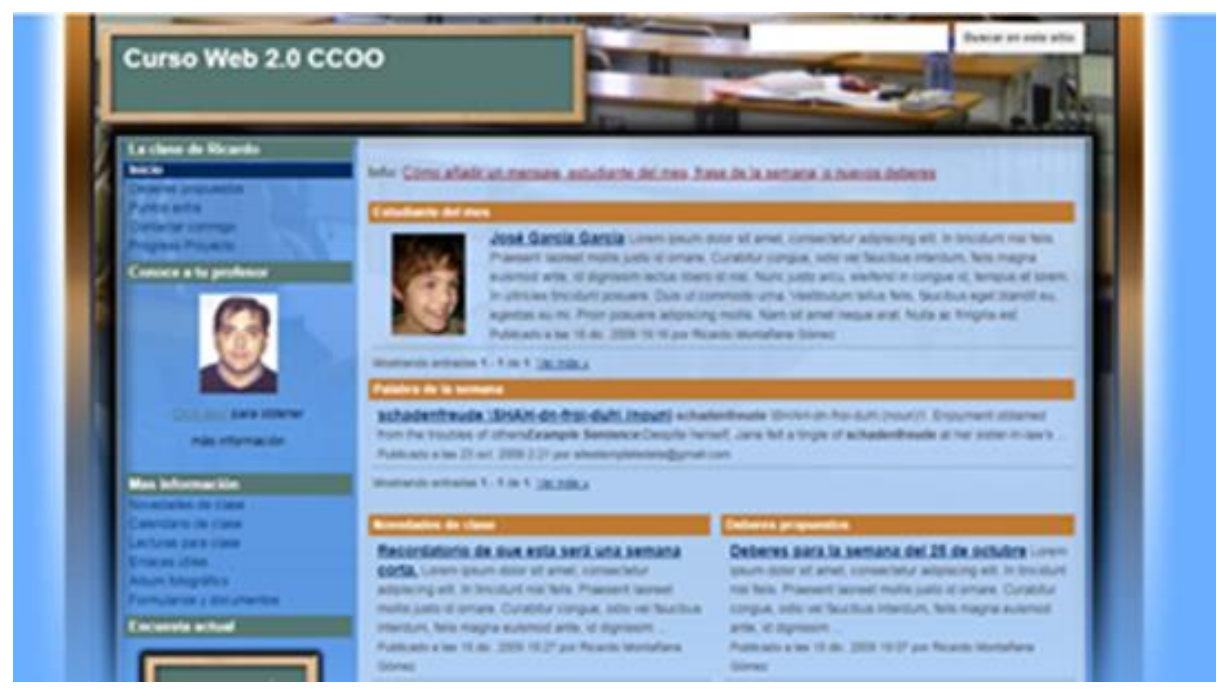

Fuente: Montañana (2017). 


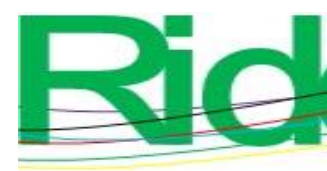

Revista Iberoamericana para la
Investigación y el Desarrollo Educativo
ISSN $2007-7467$

Fase dos

Se creó el sitio de la asignatura elegida combinando los recursos seleccionados de Google. Las herramientas que se utilizaron son las mostradas en la figura 2 (no se utilizó el chat ni tampoco el calendario).

Figura 2. Recursos de Google utilizados.

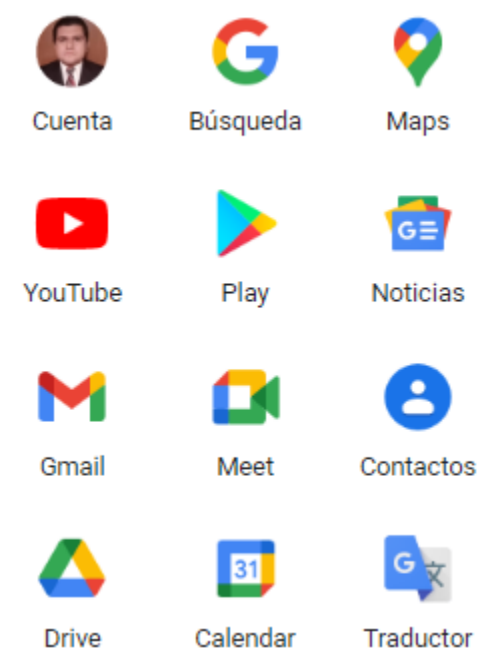

Fuente: Google (2019)

A continuación, se describe de forma breve cada uno de los recursos utilizados en la presente investigación:

- Google Site. Permite la creación de sitios web de una forma segura y dinámica.

- Google Meet. Permite la realización de videoconferencias accediendo con un código de reunión.

- Google Drive. Permite la creación de materiales, los cuales pueden ser guardados y compartidos.

- Google Documentos. Permite realizar un trabajo en línea, compartir documentos, archivos o carpetas.

- Google Hojas de Cálculo. Permite la creación, edición y colaboración de hojas de cálculo de forma individual o también de forma colaborativa.

- Google Presentaciones. Permite la creación y edición de presentaciones de una forma sencilla, colaborando con otras personas desde cualquier lugar con una conexión a internet. 

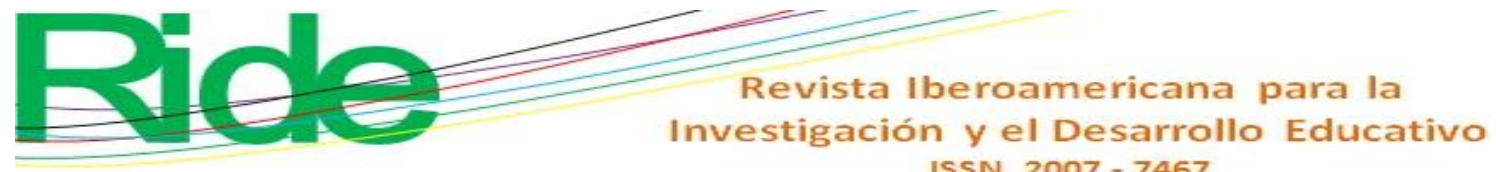

ISSN 2007 - 7467

- Google Gmail. Permite recibir y enviar mensajes; igualmente, se puede compartir información.

- Google Formulario. Permite la creación y evaluación de cuestionarios de un tópico en particular. La resolución de las preguntas es inmediata; además, permite ver los resultados parciales; arroja gráficas de cada interrogante, que se pueden descargar en una hoja de cálculo de Excel.

\section{Fase tres}

Se puso en práctica el sitio de la asignatura y la integración de los recursos de Google. A lo largo del semestre agosto-diciembre 2019, se contó con la participación y colaboración de los alumnos (ver figura 3).

Figura 3. Integración de los recursos de Google

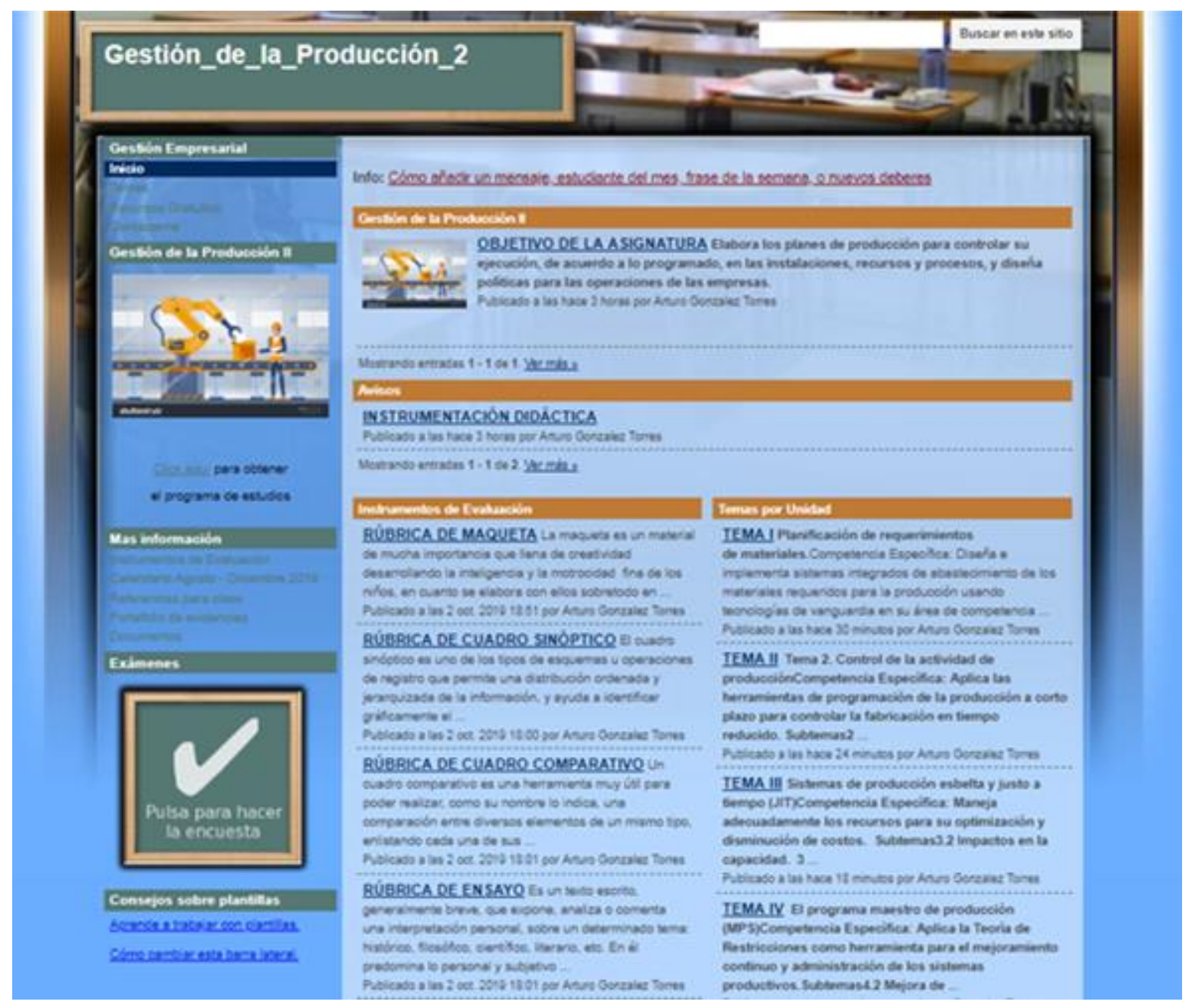

Fuente: Elaboración propia 


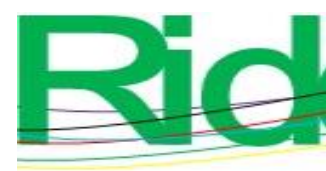

2

Revista Iberoamericana para la

Investigación y el Desarrollo Educativo

ISSN $2007-7467$

\section{Fase cuatro}

Se valoró el desempeño utilizando el instrumento de Santoveña (2010). Para ello, se utilizó la herramienta Formularios de Google, ubicada en Google Drive, y se compartió con los estudiantes para que realizaran su evaluación.

\section{Resultados}

En esta sección se presentan los resultados encontrados una vez que el proyecto logró implementarse. Así, las edades de los estudiantes fueron las siguientes: $88.88 \%$ tiene una edad de entre 17 y 19 años; $7.4 \%$ de entre 20 y 23 años, y $3.7 \%$ entre 24 y 26 años. Estas cifras también se observan en la tabla 1.

Tabla 1. Edad de los estudiantes encuestados

\begin{tabular}{|c|c|c|}
\hline Edad & Total de alumnos & \% de edad \\
\hline 17-19 años & 24 & $88.88 \%$ \\
\hline 20-23 años & 2 & $7.40 \%$ \\
\hline 24-26 años & 1 & $3.70 \%$ \\
\hline
\end{tabular}

Fuente: Elaboración propia

Como se puede observar, el rango entre 20 y 23 obtuvo un mayor porcentaje en la muestra de estudio. Respecto al género, los resultados indican que, tomando en consideración el total de la muestra unificada, los participantes del sexo masculino conforman la cifra de $44.45 \%$ y los de sexo femenino la de $55.55 \%$, tal y como se observa en la tabla 2.

Tabla 2. Género de los estudiantes encuestados

\begin{tabular}{|c|c|c|}
\hline Género & Total de alumnos & \% de género \\
\hline Femenino & 15 & $55.55 \%$ \\
\hline Masculino & 12 & $44.45 \%$ \\
\hline
\end{tabular}

Fuente: Elaboración propia

Como se puede observar, el género femenino tuvo mayor porcentaje en el número de estudiantes, el cual conformó la asignatura seleccionada para el estudio. 


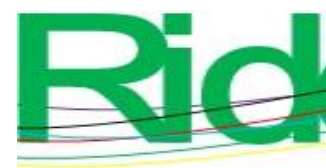

Revista Iberoamericana para la Investigación y el Desarrollo Educativo ISSN $2007-7467$

\section{Evaluación del curso virtual}

La figura 4 representa los resultados del primer factor del instrumento para evaluar el curso virtual. Se puede observar que las 17 preguntas obtuvieron un porcentaje mayor a $80 \%$. Siguiendo los resultados del ítem cinco, gracias a los recursos utilizados en la etapa de desarrollo de Google, se logró obtener una diversificación de herramientas para la materia. El promedio general del primer factor fue de $86.7 \%$, lo cual respalda que el curso virtual cumple los aspectos relativos de calidad y cantidad de contenidos didácticos.

Figura 4. Evaluación del entorno y de la metodología didáctica

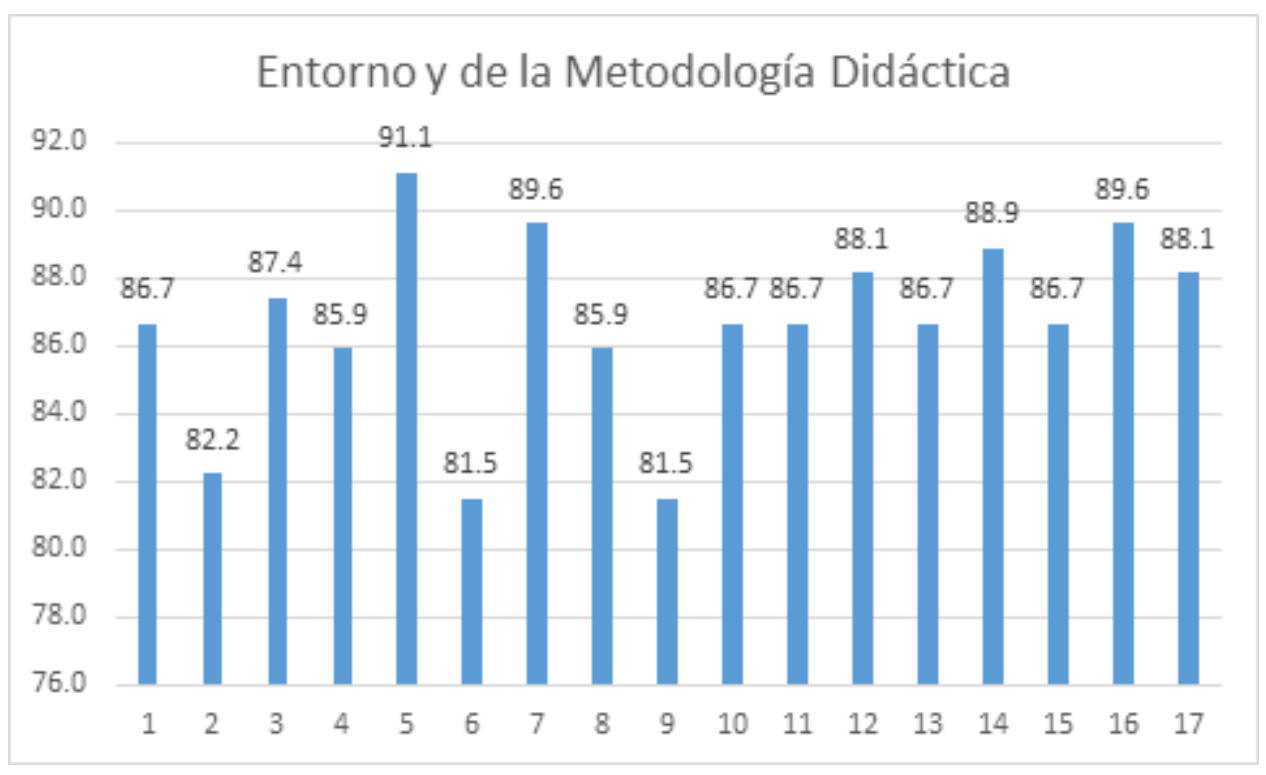

Fuente: Elaboración propia

La figura 5 representa los resultados del segundo factor de la encuesta para evaluar el curso virtual. Se puede observar que las nueve interrogantes obtuvieron un porcentaje mayor a $80 \%$. Es de destacar lo obtenido en la cuestión siete. Según los resultados de esta, adaptando las herramientas de Google, se logró obtener un diseño coherente del curso virtual. El promedio general del segundo factor fue de $83.0 \%$, lo cual deja ver que el curso virtual cumplió en ofrecer aspectos didácticos de forma completa y rica. 


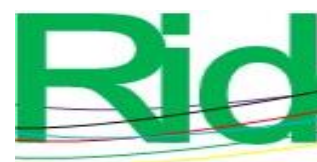

Revista Iberoamericana para la Investigación y el Desarrollo Educativo ISSN $2007-7467$

Figura 5. Evaluación de la navegabilidad y diseño

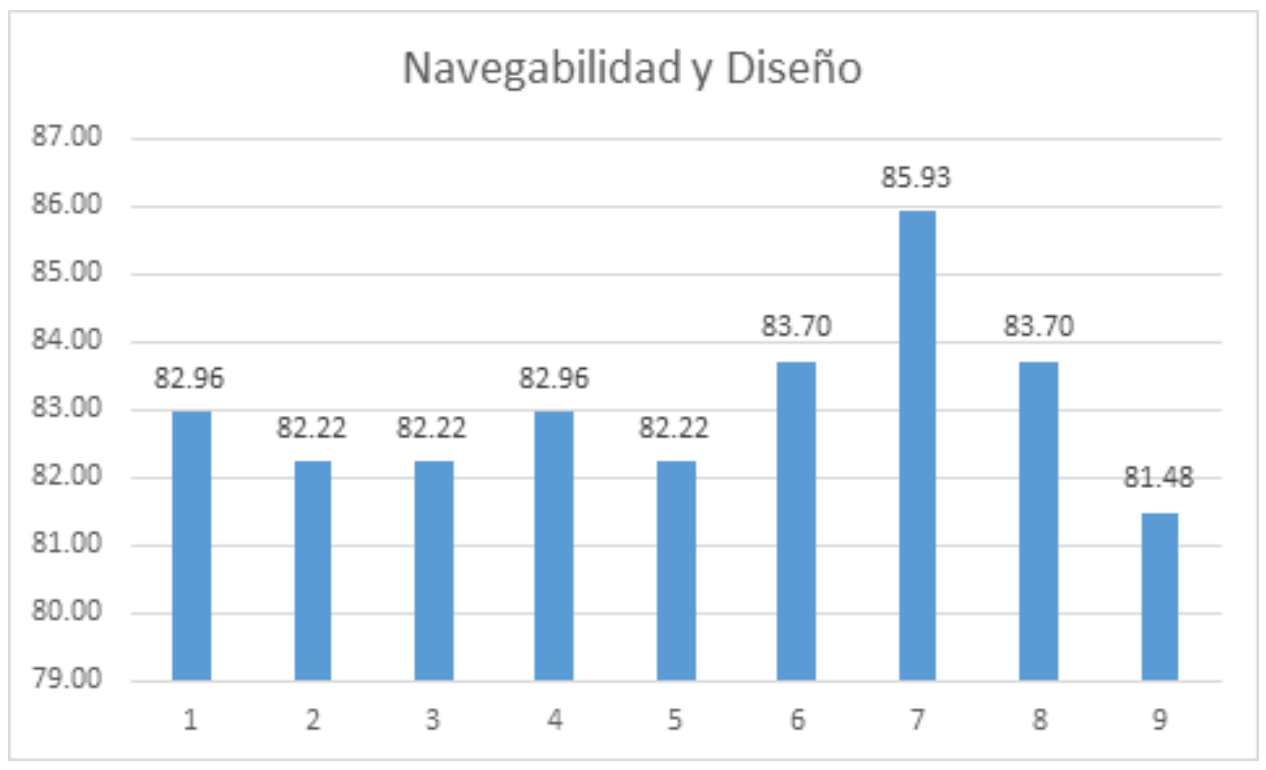

Fuente: Elaboración propia

La figura 6 representa los resultados del tercer factor de la encuesta para evaluar el curso virtual. Se puede observar que las 10 interrogantes obtuvieron un porcentaje mayor a $80 \%$. Destaca lo obtenido en la interrogante dos, ya que, adecuando los recursos de Google, se logró obtener un curso virtual que ofreció interactividad entre los estudiantes. El promedio general del segundo factor fue de $84.6 \%$, lo cual, a su vez, deja ver que el curso virtual cumplió en ofrecer funcionalidad y navegabilidad entre el alumnado.

Figura 6. Evaluación de los recursos multimedia.

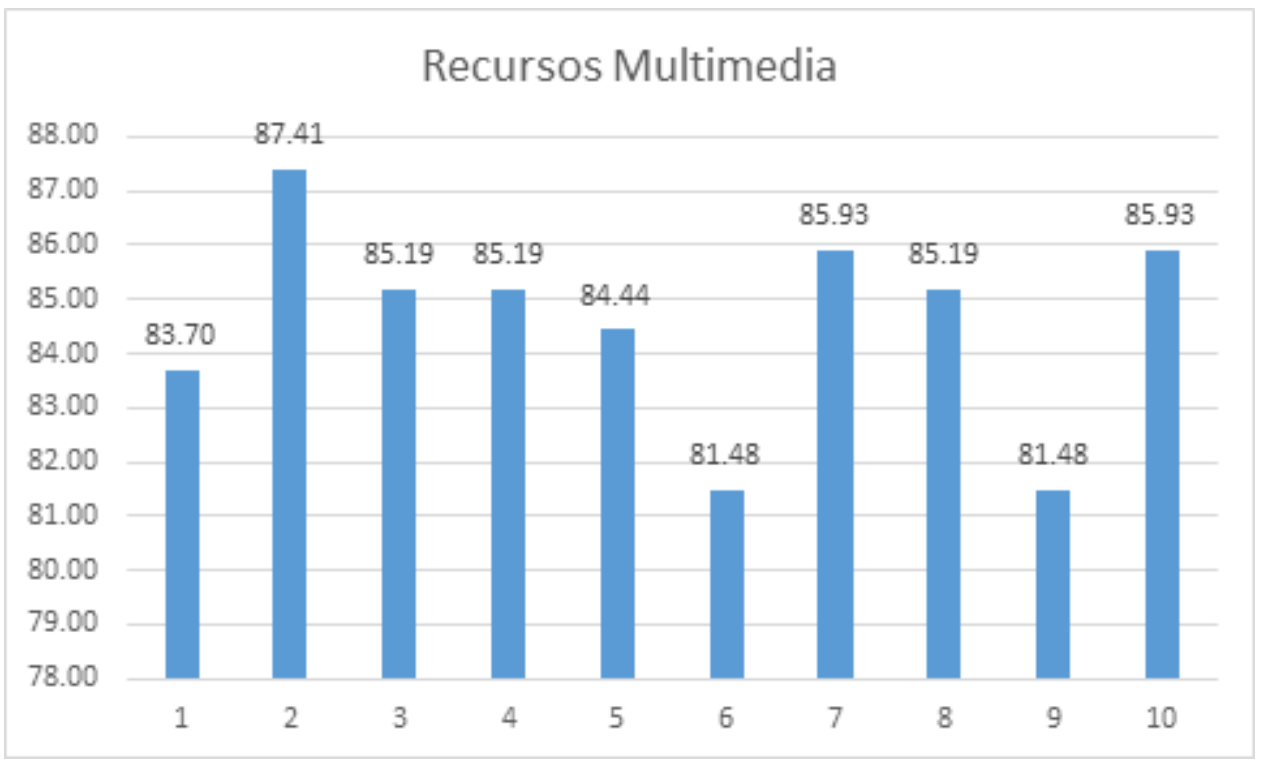

Fuente: Elaboración propia. 

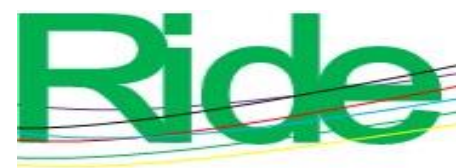

Revista Iberoamericana para la Investigación y el Desarrollo Educativo ISSN $2007-7467$

El promedio general de la percepción estudiantil sobre la evaluación del curso virtual fue de $84.6 \%$, lo cual deja ver que,se obtuvo un resultado aceptable entre la comunidad estudiantil.

\section{Aspectos de Google}

Por último, dentro de las interrogantes que se adaptaron al instrumento se muestra el resultado de la pregunta sobre el conocimiento que tenían los estudiantes acerca de los recursos de Google, la figura 7 representa los resultados del conocimiento de las herramientas de Google por parte de los alumnos.

Figura 7. Resultados del conocimiento de los recursos de Google

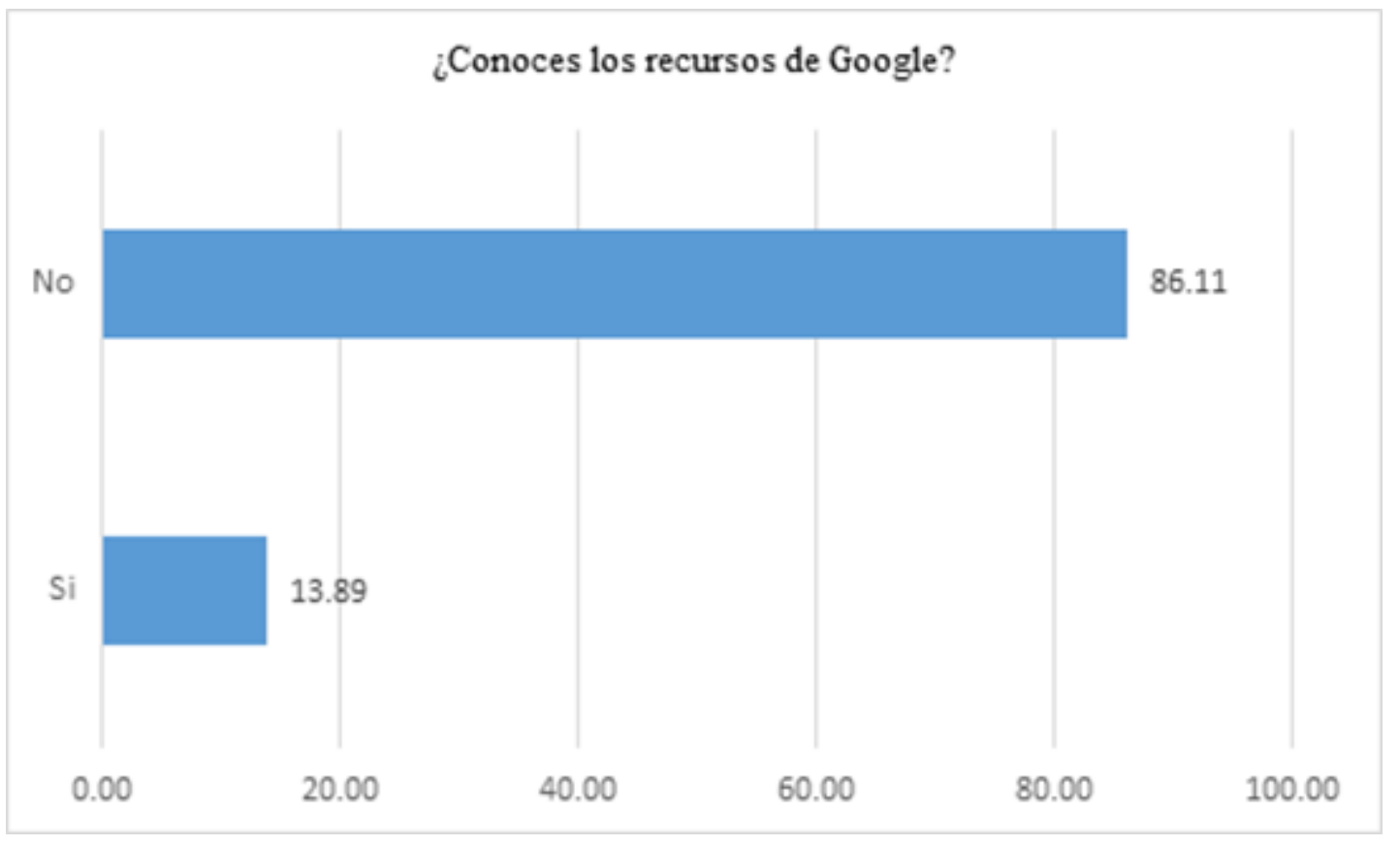

Fuente: Elaboración propia.

Se puede observar en la figura anterior que un alto porcentaje de alumnos no tenía conocimiento de los recursos de Google. Así, fue todo un reto, una prueba que se superó, demostrar a los estudiantes las ventajas que tiene el integrar dichas herramientas en el ámbito de la educación.

La evaluación de satisfacción de los recursos por parte de los estudiantes se muestra en la figura 8. Como se puede observar, los recursos más votados fueron: Formularios y Tareas (Tasks). El primero se utilizó para formular, compartir y resolver los exámenes de las unidades. El segundo se empleó para enviar e integrar las tareas que conformaban cada una 

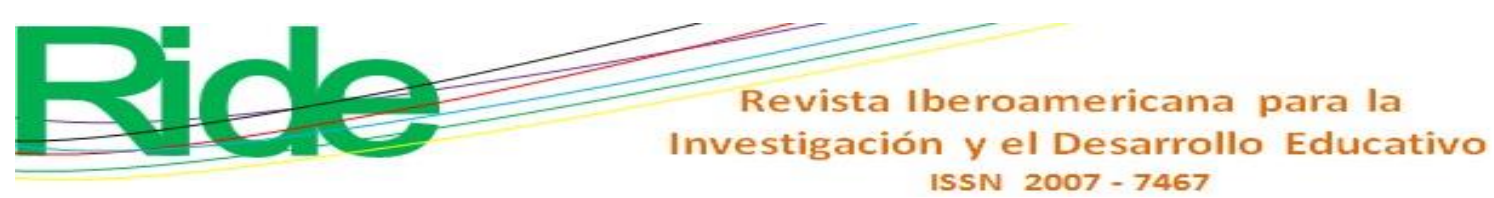

de las unidades de la asignatura. La figura 8 representa los resultados obtenidos por cada herramienta empleada en la asignatura.

Figura 8. Evaluación de satisfacción de los recursos de Google.

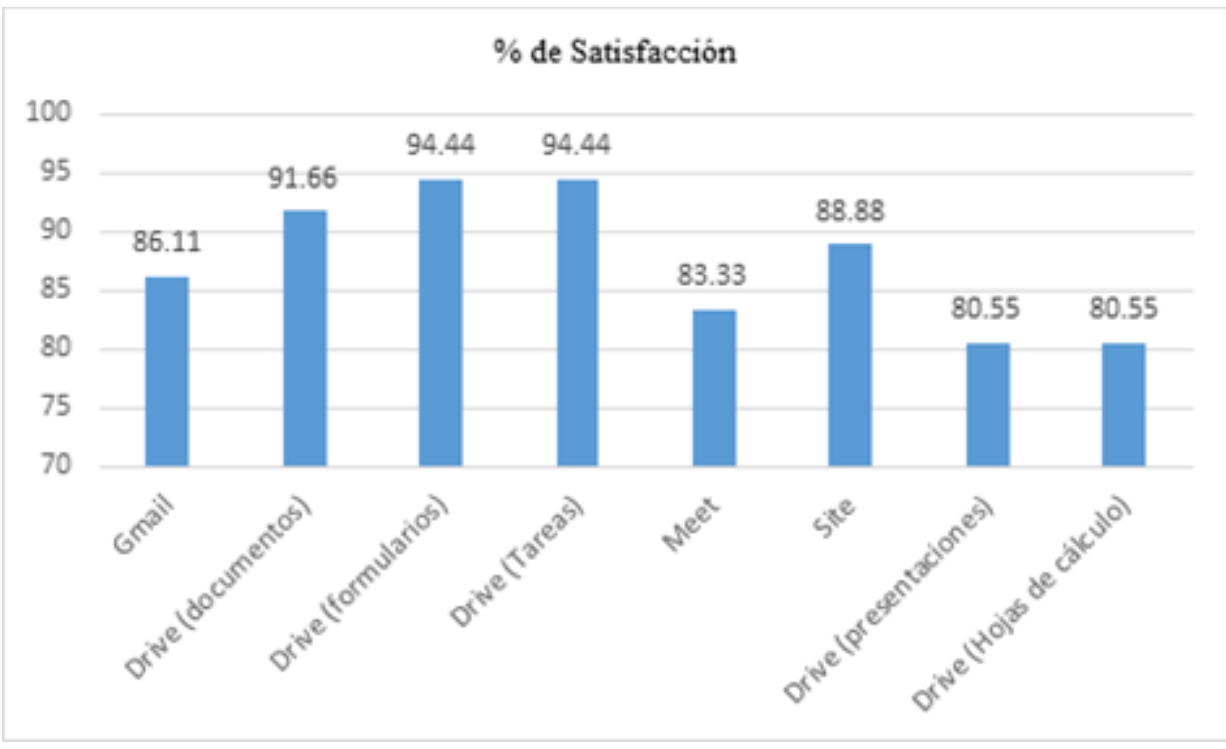

Fuente: Elaboración propia

\section{Discusión}

Una vez puesto en marcha el presente proyecto, se obtuvo información sobre cómo los estudiantes usan las herramientas tecnológicas y cómo diseñar con su ayuda actividades académicas, las cuales pueden ser desarrolladas de forma individual o de forma colaborativa.

Un dato importante fue el obtenido respecto a si los estudiantes de la asignatura en estudio conocían los recursos de Google antes de utilizarlos en clase: 86.11 \% señaló que no lo conocía, dato que coincide con los resultados de la investigación realizada por los investigadores Delgado y Casado (2013), quienes encontraron que $74.6 \%$ de los estudiantes inscritos declararon no conocer estos recursos, específicamente Google Drive. Otro hallazgo destacable es el arrojado en la evaluación de los recursos por parte de los alumnos, ya que Google Sites fue uno de los más votados; esto coincide con lo obtenido por Tolosa y García (2011) y Galantini (2015). Además, el recurso Google Drive (Tasks) obtuvo una ponderación muy elevada, lo cual coincide con los resultados obtenidos en Quinatoa (2015), Gil, Sánchez, Segura y García (2016). También, el presente estudio arrojó un alto grado de recomendación por parte de los participantes, resultado que coincide con otros estudios (Delgado y Casado, 


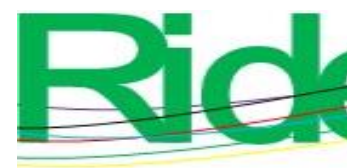

Revista Iberoamericana para la
Investigación y el Desarrollo Educativo
ISSN $2007-7467$

2013; Ruiz y Dávila, 2014). Otro descubrimiento que destacar es el beneficio de los recursos de Google en el proceso de enseñanza-aprendizaje, que igualmente coincide con otros estudios (Barrios, 2017; Castellanos y Martínez, 2013; Huzco y Romero, 2018; Zambrano, 2015). Por último, la integración de las herramientas de Google como apoyo educativo coincide con los trabajos de Garay (2012), Roda y Sassano (2016), Cabanillas y Cano (2017), Ventayen y Orlanda (2018), Kakoulli (2018) y Aparicio Arteaga (2019), todos los cuales obtuvieron resultados positivos.

\section{Conclusiones}

Los recursos que ofrece Google para el ámbito educativo son de gran utilidad, ya que ayudan a los estudiantes a desarrollar competencias basadas en el uso de las TIC. Dicho desarrollo permite estar a la vanguardia no solo en la educación, sino también cumplir con las exigencias del mercado laboral actual. Por otro lado, una característica importante de las herramientas de Google es que son gratuitas y se encuentran disponibles en línea. Además, estos recursos pueden adecuarse a las distintas necesidades educativas que puedan presentar los profesores.

Aunado a ello, un beneficio notorio al utilizar las herramientas de Google es la optimización en el tiempo, ya que facilitan muchas actividades de los alumnos. Por otro lado, el implementar los recursos de Google en la educación favorece la creación de espacios colaborativos, los cuales impactarán de una forma positiva el proceso de enseñanzaaprendizaje. Por si fuera poco, la manipulación de gran parte de estas herramientas no requiere conocimientos informáticos o de programación profundos.

El beneficio que ofrecen los recursos de Google en el ámbito educativo está probado, existen investigaciones que así lo respaldan. Lo importante será contar con una buena sinergia entre los diversos actores que estén involucrados en el proceso de enseñanzaaprendizaje. En el presente estudio se evaluaron los recursos de Google implementados en una asignatura con estudiantes de educación superior. Los resultados demostraron niveles de aceptación del uso de las herramientas altos. Cada una de ellas obtuvo una recomendación por encima de $80 \%$. Y el promedio de calificación fue de $84.6 \%$. Con la integración de los recursos de Google en la asignatura se logró poner en práctica herramientas accesibles para los alumnos, los cuales, en primera instancia, no conocían, pero conforme avanzó el ciclo escolar fueron aprendiéndolas y, al final, terminaron por aceptarlas, en su gran mayoría. En 


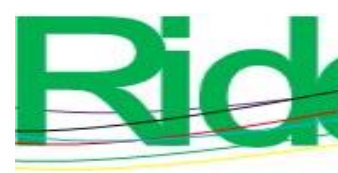

Revista Iberoamericana para la
Investigación y el Desarrollo Educativo
ISSN $2007-7467$

suma, se obtuvo un resultado más que satisfactorio en la aceptación y recomendación de herramientas de Google.

Se recomienda realizar un estudio donde se integren todos los recursos educativos de Google con el fin de aprovechar la gama y el potencial de todas las herramientas que se ofrecen. También, se sugiere capacitación para todas las partes involucradas en el proceso educativo con el propósito de ahondar más en el conocimiento y aplicación de G-Suite en el proceso de enseñanza-aprendizaje.

\section{Futuras líneas de investigación}

El presente estudio logró conocer la percepción estudiantil de la evaluación de un curso universitario integrando los recursos de Google como apoyo educativo, donde se empleó un contexto muy concreto, la asignatura de gestión de la producción II.

Una primera línea de investigación será ampliar la investigación a todas las demás asignaturas de todo el sexto semestre, en un primer momento. En un segundo momento, extender el estudio a toda la carrera de ingeniería en Gestión Empresarial. Para un tercer momento, aumentar la investigación a todos los planes y programas de estudio que el Instituto Tecnológico de Milpa Alta oferta a la comunidad estudiantil. Una vez hecho lo anterior, se podrá analizar la evolución en cada una de las ingenierías que la universidad mencionada ofrece. Además, se podrá aprender de aquellas que apliquen exitosamente los recursos de Google como apoyo educativo tanto a nivel cualitativo como cuantitativo.

Otra línea de investigación será centrarse en los profesores, indagar si estos están capacitados para el uso de los recursos de Google, qué competencias hay que fortalecer en este tópico; además, conocer si ellos promueven el uso de las TIC y de los recursos de Google a través de actividades que le permitan al alumno potencializar su aprendizaje.

Una opción más será aplicar las encuestas con alumnos de otras instituciones educativas de educación superior con el fin de conocer, mediante un estudio comparativo entre universidades, el grado (mayor o menor) de uso de las TIC y de los recursos de Google como apoyo educativo. Asimismo, conocer las estrategias de las instituciones que han logrado aplicar con éxito este paquete de herramientas en el proceso educativo. 


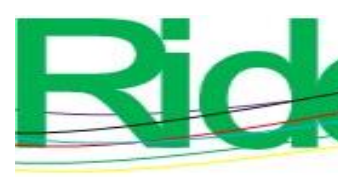
Revista Iberoamericana para la
Investigación y el Desarrollo Educativo
ISSN $2007-7467$

\section{Agradecimientos}

Un agradecimiento especial al maestro Domingo Noé Marrón Ramos, director del campus Milpa Alta del Tecnológico Nacional de México, por su apoyo en el registro y en la realización de este proyecto de investigación educativa; a la subdirección académica encabezada por el maestro Alfonso Avila Pérez Tagle, por su incondicional apoyo, y a las docentes del campus Milpa Alta Vianey Ríos Romero y Fátima Yaraset Mendoza Montero, decanas del Departamento Económico-Administrativo, por su aportación en el asesoramiento del proyecto.

Por último, agradecer a mi tutor, el Dr. Francisco Santillán Campos, por toda la ayuda prestada durante la elaboración de la presente investigación, la cual ha despertado en mí un interés creciente en el campo de la investigación educativa.

\section{Referencias}

Almeida, G. Y., Chuco, R. N. y Lavado, M. A. (2015). Herramientas de Google-Gmail y el aprendizaje del área de educación para el trabajo de las estudiantes del tercer grado de secundaria en la institución educativa Juana Alarco de Dammert-Ugel 07Miraflores-2015. (Tesis de especialidad). Universidad Nacional de Educación Enrique Guzmán y Valle, Lima.

Aparicio, C. E. (2019). Modelo sistémico de trabajo colaborativo en la nube para equipos de proyectos basado en las herramientas G-Suite, caso de aplicación: estudiantes de maestría en Dirección de Proyectos de la Universidad Nacional de Cajamarca. (Tesis de doctorado). Universidad Nacional de Piura, Piura.

Ávila, G. y Riascos, S. (2011). Propuesta para la medición del impacto de las TIC en la enseñanza universitaria. Educación y Educadores, 14(1), 169-188.

Brodersen, R. M. and Melluzzo, D. (2018). Summary of research on online and blended learning programs that offer differentiated learning options. Retrieved from https://ies.ed.gov/ncee/edlabs/regions/central/pdf/REL_2017228.pdf.

Barrios, C. D. (2017). Google Drive como herramienta pedagógica para el aprendizaje colaborativo en la asignatura Historia del nivel secundario. (Tesina de grado). Universidad Tecnológica Nacional. 


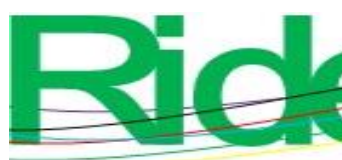

Revista Iberoamericana para la
Investigación y el Desarrollo Educativo
ISSN $2007-7467$

Barrios, I. y Casadei, C. L. (2014). Promoviendo el uso de Google Drive como herramienta de trabajo colaborativo en la nube para estudiantes de ingeniería. EDUWEB Revista de Tecnología de Información y Comunicación en Educación, 8(1), 43-56.

Botello, H. A. y Guerrero, A. (2014). La influencia de las TIC en el desempeño académico de los estudiantes en América Latina: Evidencia de la prueba PISA 2012. Ponencia presentada en el Virtual Educa 2015. Lima. Recuperado de https://vinculando.org/wp-content/uploads/kalins-pdf/singles/introduccion-ticproceso-ensenanza-aprendizaje-lengua-castellana.pdf.

Buxarrais, M. R. y Ovide, E. (2011). El impacto de las nuevas tecnologías en la educación en valores del siglo XXI. Sinéctica, (37), 1-14.

Cabanillas, M. A., y Cano, M. A. (2017). Aulas virtuales móviles utilizando herramientas $G$ Suite for Education en contraste con la intranet utilizada en la universidad de ciencias y humanidades. (Tesis de maestría). Universidad Nacional del Callao, Callao.

Castellanos, A. y Martínez, A. (2013). Trabajo en equipo con Google Drive en la universidad online. Innovación Educativa, 13(63), 75-94.

Claro, M. (2010). Impacto de las TIC en los aprendizajes de los estudiantes. Estado del arte. Santiago, Chile: Comisión Económica para América Latina y el Caribe.

Cuen, C. y Ramírez, J. L. (2013). Usos, funciones y efectos de las TIC en el aprendizaje de una licenciatura en Ciencias de la Comunicación. Ponencia presentada en el XVI Congreso Internacional Edutec 2013. San José, del 6 al 7 de noviembre de 2013.

Delgado, V. y Casado, R. (2013). Google docs: una experiencia de trabajo colaborativo en la Universidad. Enseñanza \& Teaching. Revista Interuniversitaria de Didáctica, 30(1), 159-180.

Echeverría, A. C. (2014). Usos de las TIC en la docencia universitaria: opinión del profesorado de educación especial. Actualidades Investigativas en Educación, 14(3), $1-24$.

Esparza, N. K. (2017). Percepciones de los docentes sobre el uso de las TIC en el aula: El caso de la Universidad Técnica de Babahoyo (Ecuador). 3C TIC: Cuadernos de desarrollo aplicados a las TIC, 6(1), 25-37. 


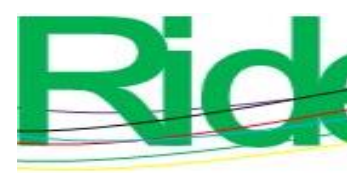

Revista Iberoamericana para la
Investigación y el Desarrollo Educativo
ISSN $2007-7467$

Galantini, J. M. (2015). Plataforma Google Site como herramienta motivadora y la organización de recursos didácticos en estudiantes de maestría. (Tesis de maestría). Instituto para la Calidad de la Educación, Lima.

Garay, V. (2012). Innovación educativa con TIC: Google Docs, una herramienta para la construcción social de conocimiento en la FID. Revista educación y tecnología, 1, 83109.

García-Valcárcel, A. y Tejedor, F. (2017). Percepción de los estudiantes sobre el valor de las TIC en sus estrategias de aprendizaje y su relación con el rendimiento. Educación $X X 1,20(2), 137-159$.

Gil, M., Sánchez, A., Segura, A. y García, O A. (2016). Cloud computing en entornos educativos online. Análisis de experiencia en la asignatura ‘Trabajo Fin de Grado’ de la Universidad Isabel. Opción, 32(11), 657-667.

Google. (2019). Ilustración de los recursos de Google. Recuperado de https://mail.google.com/mail/u/1/\#inbox.

Gómez, J. F. (2011). La utilización del Google Earth en la enseñanza de la geografía de México y del mundo con alumnos de primer grado de educación. (Tesis de maestría). Tecnológico de Monterrey, Cancún.

Gómez, M. E., Contreras, L. y Gutiérrez, D. (2016). El impacto de las tecnologías de la información y la comunicación en estudiantes de ciencias sociales: un estudio comparativo de dos universidades públicas. Innovación Educativa, 16(71), 61-80.

González, E. (2011). Recursos de Google para el desarrollo de una unidad didáctica con estudiantes de educación superior. Actualidades Investigativas en Educación, 11, 115.

Grájeda, A. F. (2015). Impacto de la utilización de la web 2.0 en el desempeño estudiantil. (Tesis doctoral). Universidad Politécnica de Valencia, Valencia.

Hernández, R. (2017). Impacto de las TIC en la educación: retos y perspectivas. Propósitos y Representaciones, 5(1), 325-347.

Hernández, R., Fernández, C. y Baptista, P. (2003). Metodología de la investigación (3. ${ }^{\mathrm{a}}$ ed.). Bogotá, Colombia: McGraw-Hill Interamericana.

Huzco, J. S. y Romero, M. F. (2018). Aplicación de las herramientas de Google Apps (Google Classroom y Google Drive) para el aprendizaje colaborativo de las alumnas del quinto año de la institución educativa CNI N 31 "Nuestra Señora del Carmen" 


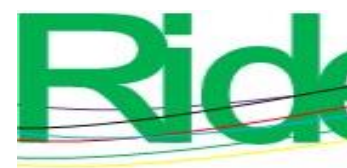

Revista Iberoamericana para la
Investigación y el Desarrollo Educativo
ISSN $2007-7467$

- Yanacancha, Pasco. (Tesis de grado). Universidad Nacional Daniel Alcides Carrión, Pasco.

Kakoulli, E. (2018). Teaching in Clouds: Using the G Suite for Education for the Delivery of Two English for Academic Purposes Courses. Journal of Teaching English for Specific and Academic Purposes, 6(2), 305-317.

Javier, A. E., Romero, L. del C. y Ricoy, C. M. (2014). El uso e impacto de las TIC en los estudiantes del nivel superior: un estudio en las carreras de Derecho y Sociología de la UJAT. Perspectivas Docentes, (50), 5-11.

López, A. E., Ledesma, R. y Escalera, S. (2009). Ambientes virtuales de aprendizaje. México: Instituto Politécnico Nacional.

Maldonado, G. M. (2014). Uso de las TIC como estrategia didáctica en el proceso enseñanza de la Geografía en 4, 5 y 6 grado de educación básica de la Escuela Normal Mixta Matilde Córdova de Sauzo de Trujillo, Colón. (Tesis de maestría). Universidad Pedagógica Nacional Francisco Morazán, San Pedro Sula.

Martínez, O. (2016). Uso de aplicaciones y plataformas online para hacer trabajos en la Universidad. Opción, 32(8), 209-224.

Mejía, G. y Gómez, R. (2017). Internet como herramienta didáctica en la formación académica en alumnos de nivel medio superior. Revista Iberoamericana de las Ciencias Sociales y Humanísticas, 6(11), 171-187.

Monsalve, M. L. (2011). Implementación de las TIC como estrategia didáctica para generar un aprendizaje significativo de los procesos celulares en los estudiantes de grado sexto de la Institución Educativa San Andrés del municipio de Girardota. (Tesis de maestría). Universidad Nacional de Colombia, Medellín.

Montañana, R. (2017). Curso Web 2.0 CCOO. Plantilla del curso. Versión clásica de Sites. Google.

Plascencia, T. N. y Beltrán, A del C. (2016). El uso de las TIC como herramienta de aprendizaje para alumnos de nivel superior. En Velasco, I. J. y Pérez, M. (coords.), Los retos de la docencia ante las nuevas características de los estudiantes universitarios. Proceedings T-XI (pp. 13-23). Tepic, México: Universidad Autónoma de Nayarit.

Quinatoa, C. (2015). Google Drive en el trabajo colaborativo de los docentes. (Tesis de grado). Universidad Estatal de Milagro, San Francisco de Milagro. 


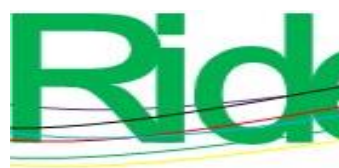

Revista Iberoamericana para la Investigación y el Desarrollo Educativo ISSN $2007-7467$

Roda, E. M. y Sassano, S. (2016). Posibilidades de Google Drive para la docencia a distancia y en el aula. Didáctica Geográfica, (16), 203-220.

Rodríguez, C. (2015). Uso de las TIC para favorecer el proceso de aprendizaje de estudiantes con discapacidad intelectual en la Institución Educativa Nicolás Gómez Dávila, Bogotá, Colombia. Estudio de caso. (Tesis de maestría). Tecnológico de Monterrey, Bogotá.

Rodríguez, R. M. (2010). El impacto de las TIC en la transformación de la enseñanza universitaria: repensar los modelos de enseñanza y aprendizaje. Teoría de la Educación: Educación y Cultura en la Sociedad de la Información, 11(1), 32-68.

Ruiz, C., y Dávila, A. (2014). Evaluación estudiantil sobre la percepción de la calidad de un curso de postgrado administrado bajo la modalidad e-learning. Compendium, 17(33), 23-42.

Santiago, G., Caballero, R., Gómez, D. y Domínguez, A. (2013). El uso didáctico de las TIC en escuelas de educación básica en México. Revista Latinoamericana de Estudios Educativos, 43(3), 99-131.

Santoveña, S. M. (2010). Cuestionario de evaluación de la calidad de los cursos virtuales de la UNED. Revista de Educación a Distancia, (25), 1-22.

Suárez, E. (2018). Uso de Google Classroom en el aula. Ponencia presentada en el Segundo Intercambio de Mejores Prácticas: TIC en el Aula. Toluca, del 6 al 8 de junio de 2018.

Tolosa, M. C. y García, J. R. (2011). Google sites como herramienta educativa. Ponencia presentada en la IX Jornadas Redes de Investigación en Docencia Universitaria 2011. Alicante, del 16 al 17 de junio del 2011.

Ventayen, R. J. M. and Orlanda, C. C. (2018). G Suite Applications in Open University System's Perspective de Google. Formamente, 13(3-4).

Vera, G. (2012). TIC en el proceso de enseñanza-aprendizaje del castellano. Revista Vinculando. Recuperado de https://vinculando.org/educacion/introduccion-ticproceso-ensenanza-aprendizaje-lengua-castellana.html\#vcite.

Vilela, J. R., Purihuamán, L. C. N. y Nuñez, B. N. (2016). Empleo de la herramienta Google Drive en la mejora de asesorías de tesis de estudiantes de Pregrado: Caso USS. Ponencia presentada en el Seminario Recursos Educativos Abiertos (REA) y MOOC.

Zambrano, M. B. (2015). Uso de las herramientas Google para la educación. (Tesis de grado). Universidad de las Fuerzas Armadas, Sangolquí. 